\title{
Nettoyage ethnique, partition et réunification à Chypre
}

Ethnic cleansing, partition and reunification in Cyprus

Ethnische Säuberung, Teilung und Wiedervereinigung in Zypern

\section{André-Louis Sanguin}

\section{OpenEdition}

Journals

Édition électronique

URL : http://journals.openedition.org/rge/548

DOI : $10.4000 /$ rge. 548

ISSN : 2108-6478

\section{Éditeur}

Association des géographes de l'Est

\section{Édition imprimée}

Date de publication : 1 janvier 2005

Pagination : 13-21

ISSN : 0035-3213

\section{Référence électronique}

André-Louis Sanguin, « Nettoyage ethnique, partition et réunification à Chypre », Revue Géographique de l'Est [En ligne], vol. 45 / 1 | 2005, mis en ligne le 18 mai 2009, consulté le 08 septembre 2020. URL http://journals.openedition.org/rge/548 ; DOl : https://doi.org/10.4000/rge.548

Ce document a été généré automatiquement le 8 septembre 2020.

Tous droits réservés 


\title{
Nettoyage ethnique, partition et réunification à Chypre
}

\author{
Ethnic cleansing, partition and reunification in Cyprus \\ Ethnische Säuberung, Teilung und Wiedervereinigung in Zypern
}

André-Louis Sanguin

\section{NOTE DE L'ÉDITEUR}

Article reçu le 17 mai 2005, accepté le 3 juin 2005

Pour l'accueil réservé et pour la documentation fournie à l'occasion de ses missions de recherche à Chypre en 1993, 1998 et 2005, l'auteur tient à remercier le Kykem (Cyprus Research Centre), la Eastern Mediterranean University (Famagouste), la University of Cyprus (Nicosie) et le Intercollege (Nicosie).

1 Lorsque l'Union Européenne ouvrit les négociations pour l'adhésion de la République de Chypre en 1997 au Sommet de Luxembourg, une hypothèse largement partagée à la fois dans les milieux de l'Union Européenne et dans les cercles scientifiques voulait que ces négociations auraient un effet catalytique sur le conflit de Chypre, en aidant à apporter une solution qui n'avait jamais pu être atteinte depuis les événements tragiques de l'été 1974, ayant eu pour effet de sectionner le pays en deux (Schoffield, 2002). À Chypre même, une opinion largement répandue voulait que, sous la pression de l'Union Européenne, une fédération finirait par voir le jour. En clair, la candidature de Chypre à l'entrée dans l'UE signifiait que le statu quo n'était plus tenable. Depuis 1974, les deux communautés, la chypriote-grecque et la chypriote-turque sont, à de très rares exceptions près, complètement séparées. De plus, depuis 1983, la population vivant au Nord de la Ligne Verte, surveillée par les Casques Bleus des Nations-Unies, se trouve sous le gouvernement de la "République Turque de Chypre-Nord» (RTCN). Jusqu'à maintenant, cet État n'a été reconnu par aucun gouvernement du monde, sauf par celui de Turquie. Différentes résolutions du Conseil de Sécurité et de l'Assemblée 
Générale des Nations-Unies ont condamné la formation de cet État sécessionniste. Dans la perspective du droit international, il n'existe qu'un État chypriote, celui fondé en 1960, et le gouvernement de cet État, bien qu'exclusivement constitué de Chypriotesgrecs, représente la totalité de l'île au niveau international. Cependant, dans les faits, ce gouvernement n'a pas de juridiction et de capacités administratives sur la RTCN.

2 Du point de vue des deux communautés, la candidature de Chypre à l'Union Européenne a changé l'équilibre politique de l'île. La situation est devenue surréaliste en avril 2004 lorsqu'à moins de deux semaines d'intervalle, deux événements considérables se sont déroulés. Le 24 avril 2004, deux référendums simultanés portant sur la proposition de réunification selon le Plan Kofi Annan se sont tenus à Chypre. Les électeurs de la RTCN dirent « oui » tandis que ceux de la République de Chypre votèrent massivement «non ». Du coup, le 1er mai 2004, seule la République de Chypre entrait dans l'Union Européenne. L'accession à l'UE n'a donc pas eu l'effet catalytique escompté ou plutôt l'a eu mais à front renversé. Malgré l'échec du Plan Kofi Annan, l'Union Européenne prend le relais de l'ONU sur le dossier chypriote en ce sens que la quasifrontière imperméable qu'est la Ligne Verte s'ouvre progressivement depuis le 23 avril 2003. Elle se transforme pour être gérée pacifiquement. Elle commence donc à être réinterprétée comme la limite entre deux entités qui deviendraient fédérées.

Jusqu'au pourrissement de la situation dans les années cinquante, Chypre constituait une merveilleuse synthèse des valeurs de l'Orient et de l'Occident et illustrait la coexistence culturelle entre les Grecs et les Turcs. C'est un peu ce paradis perdu que le grand romancier britannique Lawrence Durrell avait cherché à immortaliser dans son célèbre roman Citrons acides (Durrell, 1957). Certes, Chypre est grecque de culture et de langue depuis plus de 3000 ans mais la cession de l'île à l'Empire ottoman en 1571 par la Sérénissime République de Venise amena une population d'anciens soldats et d'immigrants anatoliens. Les Chypriotes-turcs d'aujourd'hui sont les descendants de cette population importée au XVIIe et au XVIIIe siècles. Voilà pourquoi, dans tous les déroulements de la question chypriote depuis 1955, ils n'ont jamais accepté d'être considérés comme une minorité mais comme une communauté séparée et égale en droit (Drevet, 2000). Ce sont les Britanniques qui, à partir de 1950, s'ingénièrent à saper les bases d'une vie communautaire sans tension apparente en appliquant à l'île la règle $\mathrm{du}$ « diviser pour régner » (Blanc, 2000).

\section{La période britannique (1878-1960), prélude à la partition}

Même si la plupart des analyses scientifiques sur le conflit de Chypre font remonter ses origines avant la colonisation de l'île par les Britanniques en 1878, il n'en demeure pas moins que la date de 1955 marqua la première éruption de violence sur l'île où l'on vit les objectifs politiques des deux communautés s'opposer radicalement. Que se passa-t-il pour en arriver là ? Durant toute la période ottomane (1571-1878), la majorité grecque n'avait pas eu à se plaindre du régime qui avait su associer la puissante Église Orthodoxe à l'administration publique. Cette condition valait mieux que l'humiliante subordination aux dirigeants catholiques de l'époque latine et vénitienne (1191-1571). Toutefois, la minorité turque accapara les emplois de la police et de l'administration publique. Cet état de fait continua sous le régime britannique. La majorité grecque pensait que le régime colonial britannique était provisoire et qu'il préparait l'Enosis, 
c'est-à-dire le rattachement de Chypre à la Grèce. Cette revendication monta graduellement en puissance jusqu'à la Seconde Guerre Mondiale (Drevet, 2000). Pour s'opposer au régime britannique, l'épiscopat orthodoxe fit adopter petit à petit à ses ouailles l'idée de l'Enosis. De son côté, la minorité turque ne restait pas insensible à la révolution kémaliste créant une Turquie moderne sur les ruines de l'Empire ottoman. Cela explique pourquoi fut fondée en 1943 une Turkish Minority Association. Traitant cette population européenne comme des colonisés de couleur, Londres fut incapable d'accorder l'autonomie interne à sa colonie de la Méditerranée orientale, ce qui eut pour effet de raidir encore plus la majorité grecque. L'Église Orthodoxe organisa en janvier 1950 un référendum qui révéla que $96 \%$ de la population grecque désirait l'union de l'île avec la Grèce. Cette revendication irrédentiste inquiéta la minorité turque qui craignit d'être diluée dans un grand espace hellénique, d'autant que le kémalisme lui donnait le sentiment d'appartenir à la nation turque. Le colonisateur britannique amplifia cette inquiétude en présentant l'Enosis de la majorité grecque comme un combat dirigé contre la minorité turque. En 1955, l'EOKA (organisation secrète des Chypriotes grecs) se lança dans les premières attaques contre les Britanniques afin de réaliser l'Enosis. En 1957, le TMT (organisation de défense des Chypriotes turcs) se mit en place pour répondre aux exactions de l'EOKA et s'orienta vers le Taksim (la partition), c'est-à-dire sur un projet séparatiste pour Chypre. Les années 1955-1957 virent donc l'émergence de deux représentations totalement opposées de l'avenir de l'île.

5 L'on sait que l'Empire ottoman avait quitté définitivement Chypre en 1878. De plus, son État successeur, la Turquie, avait renoncé à toute prétention sur l'île par les Traités de Sèvres (1920) et de Lausanne (1923). Le Traité de Lausanne protégeait Chypre de toute ingérence turque et Mustapha Kemal s'en était tenu à ce principe. Toutefois, le projet d'Enosis mené par la majorité grecque à Chypre réveilla en Turquie ce sentiment obsidional d'encerclement d'autant que l'archipel du Dodécanèse venait d'être rattaché à la Grèce en 1947. La Grande-Bretagne aurait pu traiter le problème de Chypre d'une manière interne. Or, pour tenter d'apporter une solution, elle choisit la voie internationale en convoquant, en août 1955, la Conférence tripartite de Londres (Grande-Bretagne, Grèce, Turquie), ce qui réintroduisait Ankara dans le jeu chypriote. Cette année-là, l'île abritait 629 villages, dont 392 purement grecs, 123 purement turcs et 114 mixtes. Depuis plusieurs siècles, les deux communautés vivaient sans friction (Sanguin, 1994, 1995). La dispersion de villages turcs sur l'ensemble de l'île était une preuve de coexistence pacifique (figure 1). 
Figure 1 :La coexistence pacifique : la dispersion des villages turcs à Chypre au moment de l'indépendance en 1960.

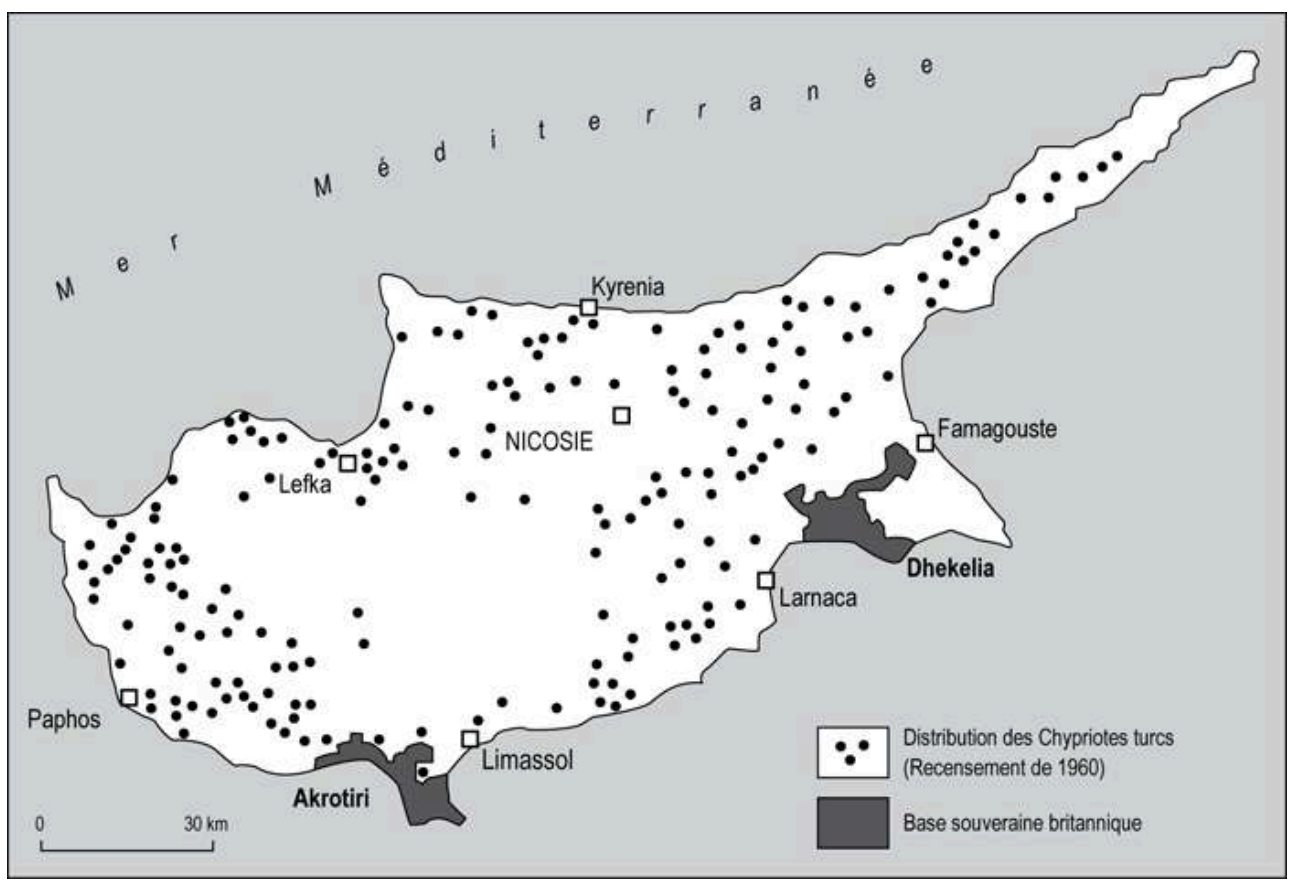

6 Les premiers affrontements intercommunautaires commencèrent en mars 1956. À ce moment, considérant la minorité turque égale en droit à la majorité grecque, Ankara demanda à l'ONU la partition de l'île. En 1958, le fossé se creusa irrémédiablement entre les Chypriotes grecs et les Chypriotes turcs à l'occasion d'affrontements intercommunautaires d'une violence inouïe. Les Chypriotes grecs isolés dans les villages et quartiers turcophones les quittèrent avec précipitation tandis que les Chypriotes turcs se mirent à fuir les villages isolés. Dépassés par l'escalade des affrontements, les soldats britanniques installèrent une ligne de démarcation entre les quartiers grecs et le quartier turc de Nicosie. La partition ethnique venait de commencer. En divisant les insulaires pour régner, les Britanniques avaient déclenché la tempête (Drevet, 2000 ; Blanc, 2000).

\section{Le premier nettoyage ethnique et le séparatisme chypriote turc (1960-1974)}

7 Le 13 août 1960, la Grande-Bretagne accorda l'indépendance à Chypre, assortie d'une Constitution agréée par les leaders des deux communautés (l'archevêque Makarios pour les Grecs comme Président de la République et le docteur Küçük pour les Turcs comme Vice-Président) et pour laquelle la Grande-Bretagne, la Grèce et la Turquie se portaient garantes. Cette constitution, boiteuse et bancale, se voulait un compromis entre la position grecque favorable à l'Enosis (rattachement à la mère-patrie) et la position turque favorable au Taksim (partition de l'île entre une zone grecque et une zone turque). Elle établissait donc un État bi-communautaire où les Chypriotes turcs étaient reconnus comme une communauté politique (et non comme une minorité!) avec des droits spéciaux qui dépassaient largement leur proportion démographique par rapport à la majorité grecque (15 sièges sur 35 au Parlement, $30 \%$ des emplois dans la fonction publique, $40 \%$ des emplois dans l'armée). Elle laissa les Chypriotes grecs déçus 
et les motifs de friction entre les deux communautés ne firent que grandir. Makarios présenta l'indépendance comme une étape vers l'Enosis, ce qui était inacceptable pour les Chypriotes turcs. Le 30 novembre 1963, Makarios proposait des amendements à la Constitution, dans un sens plus unitaire; ce que les députés chypriotes turcs du Parlement ne pouvaient accepter et, conséquemment, ils démissionnèrent en bloc du Parlement. En 1963, chaque communauté avait reconstitué ses milices. Le choc semblait inévitable. Il surgit le 21 décembre 1963 à la suite d'un incident entre policiers grecs et des civils chypriotes turcs dans Nicosie. L'embrasement se propagea à toute l'île. Les forces grecques (police et milices) isolèrent les villages et les quartiers turcs afin que ceux-ci ne puissent recevoir d'aide de l'extérieur. Ici ou là, une véritable « chasse aux Turcs » se déroula. Après cette semaine sanglante, dénommée par les Turcs kanli Noel (la Noël sanglante), le bilan s'élevait à 334 morts, en majorité turcs. Le gouvernement central n'avait rien fait pour assurer la sécurité des Chypriotes turcs. Un cessez-le-feu fut proclamé le 30 décembre et les soldats britanniques se placèrent en force d'interposition dans Nicosie et dans d'autres agglomérations. La rupture était consommée sur le plan politique et sur le plan géographique. Sur le plan politique, le gouvernement à majorité grec expulsa tous les fonctionnaires turcs qui étaient encore en poste tandis que les dirigeants de la minorité turque quittèrent toutes les institutions de la République (vice-présidence et ministères) et proclamèrent un gouvernement séparé. Par une politique d'obstruction constitutionnelle, ils se mettaient en marge de la légalité.

Victimes d'un nettoyage ethnique orchestré par les extrémistes de la majorité grecque, la minorité turque abandonna des dizaines de villages et se regroupa dans 45 enclaves où les transferts de population furent organisés par le TMT (figure 2). Fin 1964, c'était 100000 Chypriotes turcs, réinstallés dans ces enclaves, qui vivaient sous le contrôle d'une administration non reconnue par le gouvernement légal et qui porta le nom de PTCA (Administration Provisoire Chypriote Turque), financée et appuyée par la Turquie. Le Conseil de Sécurité des Nations Unies déclara ne reconnaître comme seule autorité légale de l'île que le gouvernement de la République où ne siégeait plus que des Chypriotes grecs. En mars 1964, une force de 6000 Casques Bleus des Nations Unies s'installa à Chypre et mit en place la fameuse Green Line à Nicosie (ligne de séparation entre les deux communautés dans la capitale) tout en entourant les enclaves turques afin de les protéger. L'État bicommunautaire, conçu par la Constitution de 1960, venait de voler en éclats. La partition était consommée sous le double effet du nettoyage ethnique et du séparatisme du PTCA. Sur la base d'une population turque dorénavant regroupée dans 45 enclaves, les leaders du PTCA réclamèrent une solution territoriale proche du Taksim: transformer Chypre en État fédéral avec deux communautés géographiquement séparées et installées dans des cantons ethniques. Il ne restait plus que 9000 Chypriotes turcs dans la zone gouvernementale (Patrick, 1976). 
Figure 2 : La première phase de nettoyage ethnique : le développement des enclaves chypriotesturques entre 1963 et 1974.

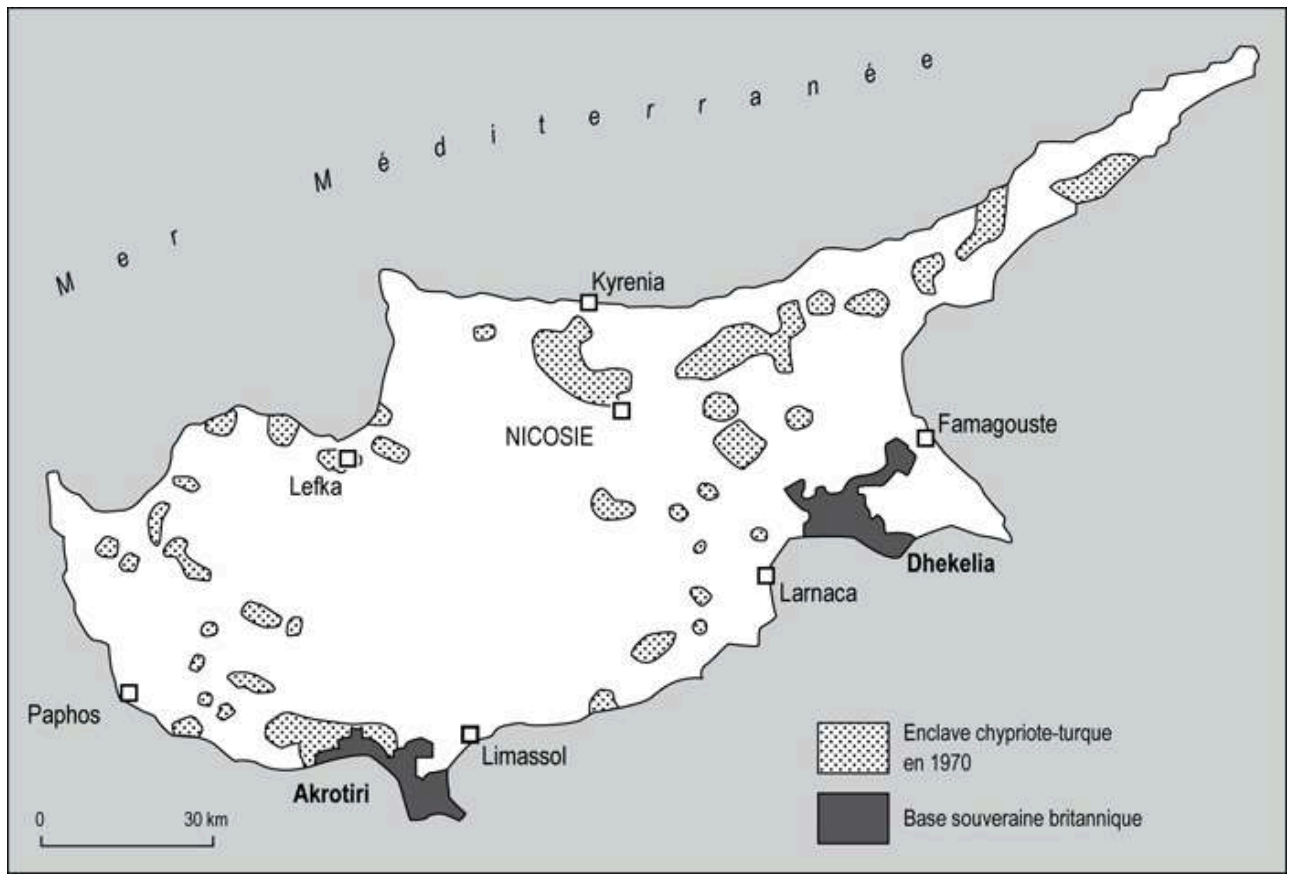

Il faut savoir que la minorité turque n'était déjà pas en position de force dans l'État indépendant de 1960. Or, ses conditions économiques empirèrent avec la partition et le séparatisme issus des événements de fin 1963-début 1964. La communauté turque n'était pas en mesure de vivre seule mais la politique de self-isolation (auto-isolement) délibérément voulue par ses dirigeants l'engagea dans une spirale de la misère: les petits agriculteurs avaient perdu leurs terres, les services publics étaient devenus d'accès difficile, l'aide internationale était filtrée par le gouvernement légal et l'argent versé par Ankara servit surtout aux dépenses de la PTCA et aux combattants des milices d'auto-défense. Nul mieux que Drevet touche du doigt ce problème: les enclaves turques étaient devenues des bantoustans à la sud-africaine dont l'unique mission était de fournir du cheap labor (main-d'œuvre à bon marché) à la zone gouvernementale en plein essor économique. Les Chypriotes turcs s'étaient donc enfermés dans un ghetto séparatiste condamné par l'ONU (Drevet, 2000). À partir de 1968, Küçük fut remplacé par Denktash, ce qui entraîna une inféodation encore plus forte de la communauté chypriote turque à Ankara. Une année auparavant, la Dictature des Colonels avait pris le pouvoir en Grèce. Ces derniers n'eurent plus qu'une idée en tête : réaliser l'Enosis, c'est-à-dire le rattachement de Chypre à la Grèce, quitte à éliminer Makarios.

\section{Les deux nettoyages ethniques de l'été 1974 et la partition des populations insulaires (1975)}

En fomentant un coup d'État contre le régime légal de la République de Chypre (le gouvernement du président Monseigneur Makarios), la Dictature des Colonels pensait obtenir immédiatement l'Enosis. C'était sans compter avec Ankara qui attendait la faute d'Athènes pour intervenir à Chypre. Après ce coup d'État des 15-17 juillet où le Président Makarios arriva à s'échapper par la base britannique d'Akrotiri, la Turquie, l'une des puissances garantes de l'indépendance de Chypre par le traité de 1960, lança 
le 20 juillet 1974 une offensive-éclair baptisée "opération de paix » qui permit de débarquer 7000 hommes à Kyrenia. De fait, le Traité de 1960 accordait un droit d'intervention pour rétablir le statu quo. En deux jours, l'armée turque avait établi un corridor avec les quartiers turcs de Nicosie (figure 3). Environ 20000 Chypriotes grecs se mirent à fuir vers le Sud. Le 22 juillet, un cessez-le-feu était imposé sur place par l'ONU. Tandis que la Dictature des Colonels s'effondrait lamentablement à Athènes, la Résolution $353 \mathrm{du}$ Conseil de Sécurité des Nations Unies demanda le retrait des belligérants sans limitation de temps. La Conférence de Genève (25-28 juillet 1974) réunit les trois puissances garantes de l'indépendance de Chypre (Grande-Bretagne, Grèce, Turquie). La Déclaration de Genève du 28 juillet reconnut quasi l'occupation turque mais surtout entérina la présence sur l'île de deux administrations séparées (la chypriote grecque et la chypriote turque). La Résolution du Conseil de Sécurité du $1^{\mathrm{er}}$ août positionna les Casques Bleus en limite de la zone conquise par l'armée turque. Début août, l'armée turque était portée à 30000 hommes dans la zone occupée.

Figure 3 : La deuxième phase de nettoyage ethnique : l'offensive-éclair et l'invasion par l'armée turque (juillet-août 1974).

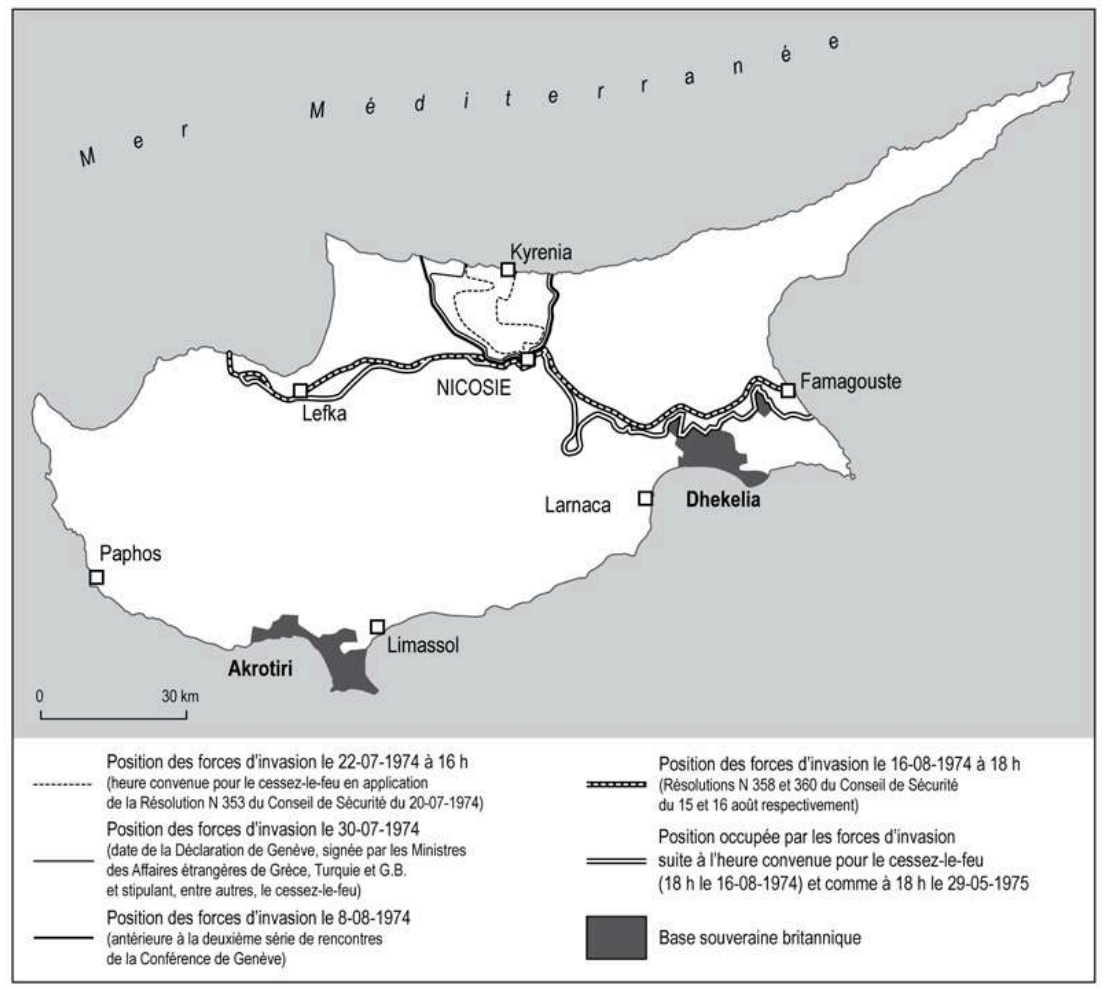

11 La deuxième offensive turque se déroula les 14-16 août 1974. L'armée turque atteignit le 35e parallèle dénommé « Ligne Attila » (figure 3). C'était la ligne de partage de l'île déjà demandée par la Turquie en 1964. Puis, après le cessez-le-feu du 16 août, les forces turques appliquèrent une tactique de grignotage, ce qui leur permit d'atteindre la bordure Nord de la base britannique de Dekhelia. En septembre 1974, la configuration territoriale de la ligne de partition, telle que nous la connaissons aujourd'hui, était atteinte. Ainsi venait de s'accomplir le deuxième nettoyage ethnique. Les deux offensives turques avaient chassé au Sud de la Ligne Attila 200000 Chypriotes grecs tandis que 60000 Chypriotes turcs se trouvaient dorénavant localisés dans la zone occupée par l'armée turque. Et les autres ? 60000 restaient bloqués dans les enclaves 
turques de 1964 demeurées au Sud et 8000 se réfugièrent dans les deux bases souveraines britanniques. L'administration chypriote turque dirigée par Denktash, appuyé par Ankara, réclama avec force leur transfert au Nord de la Ligne Attila afin de réaliser la partition définitive (Kyle, 1984 ; Petrovic, 2004).

Le résultat de la tragédie de l'été 1974 fut, à proprement parler, effroyable pour une île qui n'avait, à l'époque, que 640000 habitants : 3500 morts (3000 Grecs et 500 Turcs), 1600 disparus, 22000 hectares de forêts brûlés dans le Troodos, 200000 Chypriotes grecs déplacés au Sud de la Ligne Attila, 22000 Chypriotes turcs expulsés de leurs enclaves... Depuis 1958, la doctrine constante des dirigeants de la minorité turque avait consisté en la recherche absolue de la partition. Les deux offensives de l'armée turque (juillet et août 1974) dépassèrent largement cet objectif. En effet, à l'été 1975, la séparation géographique des deux communautés était totale (figure 4). La minorité turque se trouvait regroupée dans un espace homogène couvrant $37 \%$ de la superficie de l'île avec seulement $18 \%$ de la population insulaire, à savoir les Chypriotes turcs. Tout ce qui restait de population turcophone au Sud de la Ligne Attila (environ 20000 personnes) passa au Nord à l'automne 1975, suite à l'accord humanitaire CleridesDenktash signé à Vienne en juillet 1975. Bref, fin 1975, soit par les effets du nettoyage ethnique, soit par les effets de la partition, il n'y avait plus que des Turcs à Chypre-Nord et que des Grecs à Chypre-Sud, l'armée turque ayant interdit tout retour des Grecs en zone occupée.

Figure 4 : La troisième phase de nettoyage ethnique : partition et déplacement de population (1974-1975).

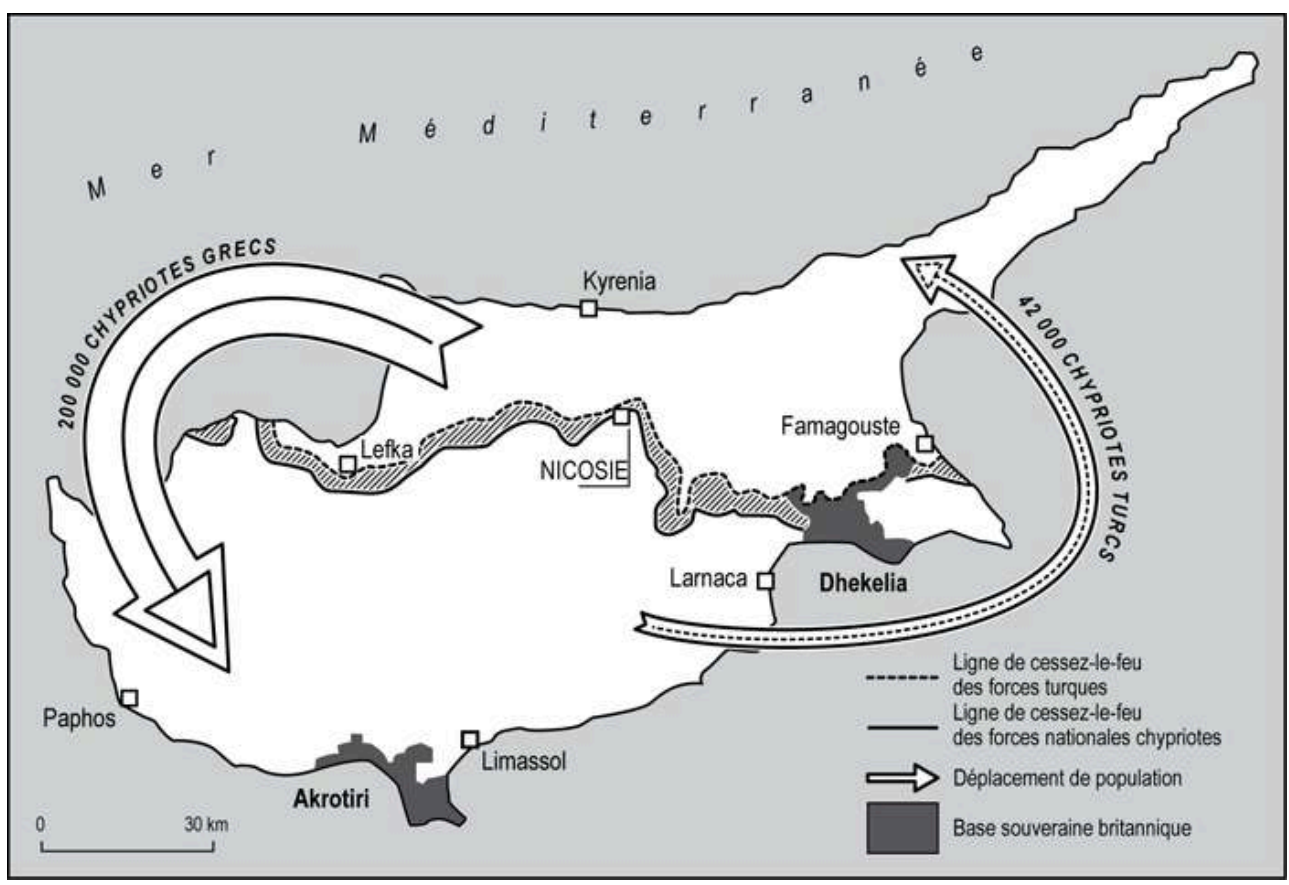

Le résultat du nettoyage ethnique fut rapidement perceptible. La majorité des réfugiés grecs vint gonfler la population de Nicosie-Sud, de Larnaca et de Limassol. Varosha, la station balnéaire de Famagouste, se transforma en ville-fantôme où errent aujourd'hui les chats et les chiens. Près de $75 \%$ des réfugiés grecs étant des ruraux, cela posa immédiatement des problèmes de reconversions sur le marché du travail. Famagouste qui avait 110000 habitants en 1974 dégringola à 55000 habitants en 1976 (tous ses 
habitants grecs l'ayant quitté). Même chose pour Kyrenia : 33000 habitants en 1973 mais seulement 23000 en 1976. Quant aux Chypriotes turcs, comme un enfant flottant dans un manteau d'adulte, le territoire où ils furent regroupés était trop grand pour eux, ce qui entraîna très rapidement la nécessité d'une immigration anatolienne. Dès l'été 1974, la Ligne Attila courrait d'une extrémité à l'autre de lîle et coupait en deux la vieille ville de Nicosie. Gardée par les Casques Bleus, elle s'étend sur $180 \mathrm{~km}$. Large de 3 à $7 \mathrm{~km}$ selon les endroits (moins de $20 \mathrm{~m}$ à l'intérieur de Nicosie), elle est ponctuée de 139 postes d'observation des Nations Unies, dont 71 occupés en permanence (figure 4). Jusqu'au déblocage de 2003, la Ligne Attila était pire que le Mur de Berlin et que le Rideau de Fer puisque les personnes, les touristes, les marchandises, les voitures individuelles et le courrier ne pouvaient passer du Sud au Nord et inversement. Les photos satellites ont bien montré que la zone tampon occupée par la Ligne Attila et les Casques Bleus représente $1 \%$ de la SAU de l'île et que ce $1 \%$ est totalement nécrosé et occupé par les friches.

Des deux côtés, les personnes déplacées et expulsées par le nettoyage ethnique et la partition ont perdu tous leurs biens. Depuis 1974, la situation n'est pas la même selon que l'on est Turc ou Grec. Au Sud, la République de Chypre gère les biens abandonnés par les Chypriotes turcs en fidéicommis, sous l'égide du Ministère de l'Intérieur. À tout moment, donc, leur rétrocession est possible à leurs véritables propriétaires. Au Nord, la RTCN a distribué tous les biens laissés vacants par les Grecs soit aux Chypriotes turcs, soit, ce qui est plus grave, aux colons importés d'Anatolie. Cette situation constitue, sans aucun doute, l'élément le plus compliqué de la résolution de la question chypriote.

\section{Statu quo pour deux États (1975-2002)}

Malgré l'amputation de quasi $37 \%$ de son territoire, la République de Chypre a pu rapidement se sortir de la catastrophe en jouant à fond la carte des échanges extérieurs : entreprises chypriotes opérant à l'étranger, exportations agricoles, zone franche, sociétés "boîtes aux lettres", pavillon de complaisance (la $6^{\mathrm{e}}$ flotte du monde en nombre de navires immatriculés), tourisme international (plus de 2 millions et demi de visiteurs par an), résidences secondaires pour étrangers... Tous ces éléments réunis, attachés à la forte valeur de la livre chypriote, ont permis à cet État amputé non seulement d'intégrer rapidement ses 200000 réfugiés qui n'ont pas eu besoin d'émigrer mais aussi d'établir une union douanière avec la Communauté Européenne en 1988, ce qui a servi de sas d'entrée pour une adhésion pleine et entière à l'Union Européenne le $1^{\mathrm{er}}$ mai 2004. La République de Chypre a également su se détacher de toute allégeance étroite avec la Grèce. L'Enosis est aujourd'hui totalement sans objet et n'est devenu qu'une matière de recherche historique! L'explication en est simple : en montant un coup d'État contre le régime légal du président Makarios, la Dictature des Colonels a précipité l'invasion turque. Pire même, lors de la seconde offensive turque d'août 1974, alors que la démocratie était revenue à Athènes, la Grèce n'a rien fait pour soutenir Chypre. Ceci est dorénavant profondément ancré dans la conscience collective des Chypriotes grecs. En d'autres mots, conscients de n'avoir à compter que sur leurs propres forces, les Chypriotes grecs se sont détachés culturellement et politiquement de la Grèce (Blanc, 2000).

Toute autre est la situation prévalant au Nord de la zone tampon tenue par les Casques Bleus. Dès février 1975, la PTCA ou «administration provisoire chypriote turque » se 
transformait en État fédéré turc de Chypre. Le sécessionnisme territorial était patent dans cette démarche politique. Puis, le 15 novembre 1983, le gouvernement de cet État non reconnu par la communauté internationale procéda à une déclaration unilatérale d'indépendance sous le titre de République Turque de Chypre du Nord (RTCN). Ceci était l'aboutissement d'une logique mise en place dès 1964 : la partition ethnique et territoriale doublée et renforcée par une politique systématique de self-isolation, c'est-àdire d'auto-isolement. Certes, cette RTCN sur la partie Nord de l'île est le résultat territorial direct de l'avancée des troupes turques à l'été 1974 et de leur nettoyage ethnique ayant chassé au Sud toute la population grecque qui se trouvait là. Mais la RTCN est aussi et encore plus l'œuvre de Rauf Denktash, leader incontesté des Chypriotes turcs depuis 1968. Denktash a été le Président élu et réélu de cette RTCN de 1983 à avril 2005. Même si le jeu démocratique s'est toujours exercé depuis 1983 (élections libres, pluralisme des partis), il n'en demeure pas moins que la RTCN a toujours été l'œuvre personnelle de Denktash.

17 À partir de 1974, les autorités de Chypre-Nord procédèrent à un panturquisme outrancier : statues et effigies omniprésentes d'Atatürk, turquisation de la toponymie, présence pesante des 35000 soldats de Turquie stationnés un peu partout, livre turque comme seule monnaie légale depuis 1983... Le problème le plus grave sans doute est cette autre forme de nettoyage ethnique, larvé et rampant, consistant à remplacer la population chypriote turque de souche par des colons importés d'Anatolie. De quoi s'agit-il ? Lors de la tragédie de 1974, on comptait 644000 habitants dans l'île, dont 497000 Grecs et 146000 Turcs. Devant l'effondrement de la situation économique en RTCN, beaucoup de Chypriotes turcs sont partis vers des cieux meilleurs et, afin de combler les vides tout en maintenant la turquité, la RTCN a fait venir des Turcs d'Anatolie, déshérités et analphabètes, à qui l'on a accordé rapidement la nationalité chypriote turque. Aujourd'hui, tout indique que la population de souche est minoritaire sur sa terre historique : 88000 personnes contre 110000 Turcs du continent sur 3350 $\mathrm{km}^{2}$.

18 La mainmise de la Turquie sur la RTCN s'explique aussi par le blocus économique dont cette dernière est l'objet. Puisque la République de Chypre est la seule autorité reconnue par la communauté internationale, la RTCN est victime d'un embargo orchestré par Chypre-Sud et rendu légal par l'ONU. Les ports de Famagouste et de Kyrenia ainsi que l'aéroport d'Ercan sont interdits à toute compagnie. Seules les compagnies turques les utilisent. Le courrier de et vers la RTCN passe par Mersin (Turquie) sous le code "Mersin 10, Turquie» car tout courrier direct est interdit d'acheminement. Conséquemment, le tourisme international fréquentant la RTCN est obligé de transiter par la Turquie. La République de Chypre refuse l'importation de tout produit en provenance de la RTCN. Au total, l'État des Chypriotes turcs se trouve dans une relation quotidienne obligée avec la Turquie, située à $70 \mathrm{~km}$ de ses côtes. Turquisation démographique et turquisation économique se complètent donc pour transformer la RTCN en une sorte de département d'outre-mer de la Turquie puisque cette dernière assure, en moyenne, $75 \%$ du budget de la RTCN (Blanc, 2000 ; Drevet, 2000). 


\section{Le déblocage de 2003-2005 : la fin de la ligne de séparation et la réunification en perspective}

19 Ainsi, depuis les tragiques événements de l'été 1974, la situation semblait complètement et pour longtemps figée à Chypre. Était-il imaginable de spéculer sur une coupure définitive de l'île en deux? Pouvait-on imaginer deux États tournés dos à dos comme Haïti et la République Dominicaine sur l'île d'Hispaniola ? Que se passa-t-il pendant quasi trois décennies? Au Nord, le régime Denktash poursuivait, coûte que coûte, sa politique d'auto-isolement, de «bunkérisation » et de relation unique avec la Turquie au point d'en être le vassal. Au Sud, la République jouait la carte de l'arrimage à l'Europe avec en ligne de mire l'adhésion et l'entrée dans l'Union Européenne. Arriva l'année 1990, celle de la réunification de l'Allemagne. Elle fut un révélateur pour les dirigeants chypriotes car elle montra ce que pouvaient être les coûts pour mettre à niveau la partie la moins avancée en termes économiques (il convient de rappeler que, de 1990 à 2005, l'ex-RFA a injecté 1500 milliards d'euros dans l'ex-RDA pour la mettre à niveau). L'accession de Chypre à l'Union Européenne a été considérée par les deux gouvernements de l'île de deux façons apparemment contradictoires. La première fut de percevoir cette accession comme une solution au conflit chypriote dans la mesure où le nouveau statut de Chypre comme membre de l'Union aurait plus d'importance que la division ethnique. La seconde fut de considérer cette accession comme tout simplement « illégale » dans la mesure où elle se superposerait à la Constitution de 1960. Or, cela aurait requis l'accord des deux communautés de l'île avant que l'État chypriote puisse se joindre à un autre État. Dans cette seconde perspective, l'union avec l'Europe aurait signifié la «solution » parce qu'elle aurait provoqué l'union de la RTCN avec la Turquie, après quoi le problème de Chypre aurait été résolu. Quels que soient les arguments à l'appui ou à l'encontre de ces deux positions conceptuelles, la pratique a montré que la division de facto de Chypre et son unité de jure pouvaient être compatibles avec l'adhésion à l'Union Européenne. La République de Chypre considère qu'il n'y a pas violation de la Constitution de 1960 puisque l'Union Européenne n'est pas un État et n'est pas dotée de la personnalité juridique. C'est pourquoi le Conseil Européen déclara en 1995 le bien-fondé de la candidature de Chypre. Les négociations commencèrent donc en 1997 pour se conclure en décembre 2002. Le Traité d'Accession fut signé en avril 2003 pour prendre effet au $1^{\text {er }}$ mai 2004.

$\mathrm{Au}$ Nord, deux changements politiques ont très fortement contribué à changer radicalement la donne politique : le virage pro-européen du Gouvernement Erdogan en Turquie et la lassitude de Chypriotes turcs face au régime Denktash. Pour la première fois dans l'histoire récente de la Turquie, une formation politique, à savoir le parti AKP, a obtenu quasi les deux tiers des sièges au Parlement à Ankara en 2003. Son leader, Recep Tayyip Erdogan, est devenu Premier Ministre avec un mandat et un programme clairs : faire entrer la Turquie dans l'Union Européenne. En vertu du dicton « Paris vaut bien une messe ", Ankara a effectué, en moins de deux ans, un virage à $180^{\circ}$ sur la question chypriote et cela selon deux modalités : d'une part en favorisant le dossier de la réunification de Chypre et en appuyant le Plan Kofi Annan de réunification; d'autre part, en lâchant le régime Denktash et sa self-isolation sans issue. Dès le 23 avril 2003, la Ligne Verte était réouverte au "Ledra Checkpoint» au cœur de Nicosie. Pour la première fois depuis 1974, les Chypriotes pouvaient se rendre de l'autre côté de la ligne de séparation. Le 14 décembre 2003 avaient lieu des élections législatives en RTCN qui 
virent, pour la première fois, une coalition de partis anti-Denktash, pro-réunification et pro-Europe arriver au pouvoir avec comme Premier Ministre Mehmet Ali Talat. À partir de cette date, toutes les parties concernées par le dossier (République de Chypre, RTCN, Grèce, Turquie, Union Européenne) s'accordèrent pour trouver une solution à la question chypriote avant le $1^{\text {er }}$ mai 2004. La pression vint aussi de la propre population chypriote turque, excédée par quasi quatre décennies d'anatolisation à outrance. Sur 198000 habitants en 2005, la RTCN n'abrite plus que 88000 Chypriotes turcs de souche, devenus minoritaires sur leur propre terre ancestrale et historique. Il y a là, d'ailleurs, un curieux processus de «nettoyage ethnique dans le nettoyage ethnique ». Pendant longtemps, Denktash a pu se maintenir au pouvoir en jouant sur l'insécurité de ses concitoyens, sur le rôle protecteur de l'armée turque et sur une attitude antieuropéenne. Le cul-de-sac économique dans lequel il a entraîné la RTCN a développé chez les Chypriotes turcs un fort sentiment d'« insularité dans l'insularité ». Au fur et à mesure que la République de Chypre se rapprochait de l'Union Européenne, les Chypriotes turcs ont bien vu que la roue de l'Histoire avait tourné et qu'ils risquaient de rester sur le bas-côté de la route. Une opposition s'est donc levée contre ce système sclérosé. La réunification a donc été vue comme un moyen pour sortir de la stagnation économique de la RTCN, pour stopper l'émigration des Chypriotes turcs de souche, pour endiguer l'immigration quasi continue de colons anatoliens analphabètes et peu qualifiés et, enfin, pour développer le fantastique potentiel touristique de Chypre-Nord.

21 Afin d'accélérer le processus de règlement du conflit chypriote avant la date fatidique du $1^{\mathrm{er}}$ mai 2004, Kofi Annan, Secrétaire Général des Nations Unies, reprenant et développant le set of ideas établi en 1992 par son prédécesseur Boutros Ghali, présenta en 2002-2003 un plan de réunification de Chypre. Dans ses paramètres les plus territoriaux, le Plan Annan prévoyait deux États constituants (chypriote grec et chypriote turc) de statut identique dans une organisation confédérale où l'essentiel des compétences aurait résidé dans les entités constituantes. Le territoire chypriote turc aurait été réduit de 37 à $28 \%$ de la superficie de l'île (Morphou et Varosha étant rendues aux Grecs). La moitié des quelque 110000 colons turcs immigrés depuis 1974 seraient retournés en Anatolie tandis que 123000 des 200000 réfugiés grecs de 1974 auraient été autorisés à retourner dans leurs foyers au Nord de la Ligne Verte. Le Plan ne prévoyait ni armée fédérale ni armée des États constituants mais la présence de 6000 soldats de Grèce et de 6000 soldats de Turquie et le maintien des Casques Bleus pour la mise en œuvre du plan de réunification jusqu'en 2011. Le Plan Annan fut soumis à référendum dans les deux parties de l'île le 25 avril 2004, soit une semaine avant l'entrée de Chypre dans l'Union Européenne, ce qui ne constituait certainement pas un bon calendrier ! Le résultat fut tout à fait étonnant : $65 \%$ de « oui » en RTCN mais $75 \%$ de «non » en République de Chypre. Le refus de Chypre-Sud fit que, une semaine plus tard, seule la République de Chypre, telle que réduite territorialement depuis 1974, entra dans l'Union Européenne. Cette République de Chypre s'étend sur $5900 \mathrm{~km} 2 \mathrm{de}$ l'île et abrite, en 2005, 703000 habitants. Le « oui » massif en RTCN s'est expliqué par le désir de sortir de l'isolement, de rentrer dans la dynamique de l'Union Européenne et de participer à sa prospérité économique. En revanche, le «non » massif exprimé en République de Chypre a eu plusieurs motivations entrecroisées: crainte d'une réunification "à l'allemande " aux coûts exorbitants, non reconnaissance de la République de Chypre par la Turquie, surface du territoire de l'entité constituante chypriote turque disproportionnée par rapport au nombre réel de la population chypriote turque, maintien en partie Nord de la moitié des colons anatoliens importés 
depuis 1974 et considérés comme "illégaux " par les autorités de la République, retour de seulement 123000 et non des 200000 réfugiés grecs victimes de l'invasion turque de 1974, présence des 30000 soldats turcs stationnés au Nord.

Aux élections législatives du 20 février 2005 en RTCN, le parti du Premier Ministre sortant Mehmet Ali Talat et les formations associées remportèrent la majorité des sièges au Parlement de Nicosie-Nord. Puis, à l'élection présidentielle du 17 avril 2005 en RTCN où Denktash ne se représentait pas, Talat a été élu, dès le premier tour, sur un clair programme pro-européen de réunification. La balle est désormais dans le camp de la République de Chypre qui continue à ignorer la RTCN en cherchant à l'enfermer et à l'isoler, alors que, d'une part, l'Union Européenne s'emploie à rouvrir un maximum de points de passage le long de la Ligne Verte et que, d'autre part, elle met en place en RTCN des programmes de développement en vue de la mettre à niveau avec la partie Sud (Hocknell, 2001). Il est clair qu'après le fiasco retentissant du Plan Kofi Annan en avril 2004, c'est maintenant à l'Union Européenne de prendre le relais pour relancer le processus de réunification. «Vivre ensemble séparément " peut tout à fait constituer le fil conducteur d'une réunification de l'île d'Aphrodite qui semble inéluctable, tant l'Union Européenne ne pourra supporter pendant des décennies une plaie purulente sur sa frontière la plus extrême en Méditerranée orientale.

\section{BIBLIOGRAPHIE}

Blanc P. (2000). - La déchirure chypriote. Géopolitique d'une île divisée, Paris, L'Harmattan, 288 p.

DREVET J.-F. (2000). - Chypre en Europe, Paris, L'Harmattan, 328 p.

DuRRell L. (1957). - Citrons acides, Paris, Buchet-Chastel.

HoCKNELl P. (2001). - Boundaries of Cooperation: Cyprus de facto Partition and the Delimitation of Transboundary Resource Management, New York, Aspen Publishers.

KYLE K. (1984). - Cyprus, London, Minority Rights Group Publications.

PATRICK R. (1976). - Political Geography and the Cyprus Conflict, Waterloo, Waterloo University Press.

Peristianis N. (1998). - «A Federal Cyprus in a Federal Europe », Cyprus Review, Tome 10, n 1, pp. $33-43$.

Petrovic D. (2004). - « Ethnic Cleansing, An Attempt at Methodology », European Journal of International Law, Tome $\mathrm{V}, \mathrm{n}^{\circ}$ 3, pp. 342-359.

Pratt M., BRoWn J. (2000). - Borderlands under Stress, New York, Aspen Publishers.

SANGUIN A.-L. (1994). - « Chypre, les dimensions ethniques d'une partition et l'avenir d'un pays ", Cahiers de Géographie du Québec, Tome 38, n²104, pp. 165-184.

SANGUIN A.-L., DOLGAN-PETRIC M. (1995). - « Ciper, Negotova Prihodnost Afroditinega Otoka » (Cyprus, The Uncertain Future of the Island of Aphrodite), Geografski Obzornik, Tome 42, n², pp. 12-17. 
SCHOFFIELD C., NEWMAN D., DRYSDALE A., BROWN J. (2002). - The Razor's Edge : International Boundaries and Political Geography, New York, Aspen Publishers.

\section{RÉSUMÉS}

La fin de la période coloniale britannique à Chypre a marqué le prélude à la partition entre la communauté grecque et la communauté turque. Puis, les premières années de l'indépendance de la République de Chypre ont vu s'accentuer le séparatisme turc, conséquence d'un nettoyage ethnique orchestré par la communauté grecque. L'invasion de Chypre par l'armée turque en 1974 a entraîné un second nettoyage ethnique, cette fois-ci, aux dépens de la majorité grecque. Dès 1975, la partition des populations insulaires était consommée par la mise en place de la Ligne Verte, gardée par les Casques Bleus des Nations-Unies. De 1975 à 2002, régna le statu quo entre deux États : au Sud, la République de Chypre, seule reconnue par la communauté internationale ; au Nord, la République Turque de Chypre-Nord (RTCN), État vassal d'Ankara, uniquement reconnu par la Turquie. Un déblocage de la situation est intervenu en 2003 lorsqu'un gouvernement pro-européen est arrivé au pouvoir au Nord et, en 2004, lorsque la République de Chypre est entrée dans l'Union Européenne. La fin de l'ère Denktash en avril 2005 et l'arrivée d'un président pro-réunification en RTCN changent radicalement une donne géopolitique complètement figée depuis trente ans. La réunification de l'île d'Aphrodite relève-t-elle dorénavant de l'ONU (malgré l'échec du Plan Kofi Annan en 2004) ou davantage de l'Union Européenne?

The end of the colonial British period in Cyprus emphasized the prelude to the partition between the Greek community and the Turkish one. Then, the first years of the independence of the Republic of Cyprus saw to become accentuated the Turkish separatism, as a clear consequence of an ethnic cleansing organized by the Greek community. The invasion of Cyprus by the Turkish Army in 1974 generated a second ethnic cleansing, this time, at the expense of the Greek majority. From 1975, the partition of island's communities was accomplished by the implementation of the Green Line, monitorized by the UN Blue Helmets. From 1975 to 2002, a statu quo was prevailing between both States : in the South, the Republic of Cyprus, single State recognized by the international community; in the North, the Turkish Republic of Northern Cyprus (TRNC), Ankara's puppet State, solely recognized by Turkey. A freeing of the situation emerged in 2003 when a pro-EU government came into office in the North and when the Republic of Cyprus entered EU in 2004. The end of the Denktash era in April 2005 and the arrival of a proreunification president in TRNC radically changed the geopolitical deal which was totally frozen since thirty years. Does henceforth the reunification of the «Island of Aphrodite " come under UN (despite the fail of the Kofi Annan Plan in 2004) or more under EU?

Das Ende der britischen Kolonialzeit in Zypern bestimmte das Vorspiel zur Teilung zwischen der griechischen und der türkischen Gemeinschaft. Dann wurde in den ersten Jahren der unabhängigen Republik Zypern der türkische Separatismus deutlich, Folge einer ethnischen Säuberung, inszeniert durch die griechische Gemeinschaft. Die Invasion Zyperns durch die türkische Armee 1974 hat eine zweite ethnische Säuberung mit sich gebracht, diesmal auf Kosten der griechischen Majorität. Seit 1975 wurde die Teilung der Inselbevölkerung durch die Einrichtung der Grünen Linie vollzogen, bewacht durch die Blauhelme der Vereinten Nationen. Von 1975 bis 2002 herrschte der Status quo zwischen zwei Staaten: im Süden die Republik Zypern, allein anerkannt durch die internationale Gemeinschaft, im Norden die türkische Republik Zypern Nord, Vasallenstaat von Ankara anerkannt nur durch die Türkei. Eine Entkrampfung der Lage zeichnete sich 2003 ab, als eine proeuropäische Regierung im Norden zur 
Macht kam und 2004, als die Republik Zypern in die Europäische Union eintrat. Das Ende der Ära Denktash im April 2005 und der Auftritt eines zur Wiedervereinigung neigenden Präsidenten im Norden änderten radikal die seit 30 Jahren eingefrorene politische Lage. Die Wiedervereinigung der Insel der Aphrodite - wird sie mehr von der UNO (trotz des Scheiterns des Plans von Kofi Annan 2004) oder mehr von der EU bestimmt werden?

\section{INDEX}

Schlüsselwörter : Ethnische Säuberung, Europäische Union, Griechenland, Politische Geographie, Teilung, Türkei, Wiedervereinigung, Zypern

Keywords : Cyprus, ethnic cleansing, European Union, Greece, partition, political geography, reunification, Turkey

Mots-clés : Chypre, géographie politique, Grèce, nettoyage ethnique, partition, réunification, Turquie, Union Européenne

\section{AUTEUR}

\section{ANDRÉ-LOUIS SANGUIN}

UFR de Géographie, Université de Paris IV-Sorbonne, 191 rue Saint-Jacques, F-75005 Paris, France, alsanguin@wanadoo.fr 